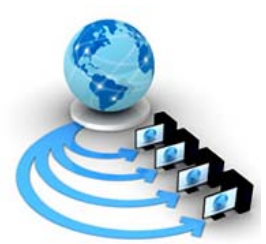

\title{
ENHANCED ROUTE SELECTION APPROACH OF GPSR USING MULTIPATH FORWARDING IN VANET
}

\author{
Hitesh Patel, Ph.D. Scholar \\ Computer Science \& Engineering \\ Rai University \\ Ahmedabad, India
}

\author{
Dr. G. R. Kulkarni, Principal \\ S.S. Agrawal Institute of Engi. \& Tech. \\ Gujarat Technological University \\ Navsari, India
}

\begin{abstract}
The Vehicular Ad-Hoc Network is infrastructure less network in which vehicles are connected without wires. Routing in Vehicular Ad-hoc Network is challenging nowadays due to increased no of a vehicle, the high mobility of nodes, dynamically changing topology and highly partitioned network, so the challenges on the roads are also increased like roads are full, the safety problem, speed etc. GPSR is a position based algorithm. GPSR uses source node and destination node's position to forward packets. This protocol uses Greedy Forwarding and Perimeter Forwarding strategies for packet forwarding from source to destination. The proposed system uses the modify beacon packet strategy which includes source node and destination node parameters to route packet. All nodes are initially selected as forwarding node which is a letter on reduced to final routing path after acknowledgment. The proposed system improves the parameters like Packet Delivery Ratio and maintains a number of hop counts.
\end{abstract}

Keywords: VANET, GPSR, Routing Protocols, Route Selection, Greedy forwarding, Perimeter forwarding,

\section{INTRODUCTION}

Vehicular ad hoc networks (VANETs) are one of the types of MANETs that are formed between various moving vehicles. In VANET each and every node are joining the network and leaving the network at any time. Each node is capable of sending and receiving the packets. Various nodes are located at a different location but it is not necessary that they are adjacent to each other. Therefore, routing can be done between two nodes and uses the multi path mechanism for sending and receiving the packets. When VANET environment is implemented using popular MANET routing protocols for simulation, it results shows that, all the protocols have large packets overhead because the routes changes very frequently due to high mobility of nodes. So we need the enhanced routing protocols for VANET based on its characteristics and issues.

Several routing protocols have been proposed to achieve high PDR, minimum delay, storage, bandwidth and exchange of control information. In Direct Delivery protocols source node carry message until it find destination node. First contact routing routes to first node it encounter as random walk to search for destination.

The NDTN Routing Protocols are also known as Min delay protocols. The protocols always trying to minimize the packet delivery time and increase PDR from source to destination by trying to find the shortest path. NDTN protocols again classified in to beacon based, non-beacon based and hybrid routing protocols. Here is the Issues for NDTN routing protocols [1,2]:

- $\quad$ As the NDTN routing always try to reduce the packet delivery time. Packets always pass through intermediate nodes with shortest and optimal path to achieve this.

- Neighbour knowledge is required to find the shortest path between nodes.

- Recovery mechanism is required if packet will loss during routing.
In VANET, The position based routing protocols is used more frequently than other. GPSR is using greedy forwarding technique in NDTN routing protocols. In this approach packets are forwarded to a neighbor who is geographically closer to the destination node.

The beacon based routing protocols use the "HELLO" beacon message to search for neighbors. The node sends the beacons for regular interval to maintain the neighbor lists. It again classified into non-overlay and overlay. Like GPSR, PBR-DV, GRANAT, DGR, GPCR, GPSRJ+, CAR, GSR, ASTAR, STBR, GyTAR, LOUVRE.

Rest of the paper is organized as follow. Section II describes various routing protocols along with GPSR. In section III, we have provided proposed routing protocol algorithm. Performance parameter and Simulation results are shown in Section IV and V. Section VI and VII discuss conclusion and future works. Finally Section VIII list references used for research of this paper.

\section{PRELIMINERIES}

\section{A. Related Works}

A-STAR-Anchor-based Street-and Traffic- Aware Routing is having consciousness of the physical environment around the vehicles; the protocol can take wiser routing decisions [22]. It consists of including within the packet header the list of junctions (anchors) that the packet must traverse. A-STAR depends on GSR. A-STAR provides inclusion of traffic density information to weigh the streets of the scenario.

CAR- Connectivity-Aware Routing algorithm is divided into three stages: (i) finding the location of the destination node and path to reach destination node, (ii) using that path to transmit messages, and (iii) maintaining the connectivity between nodes due to the mobility of vehicles [23].

GPCR forms a planar graph such that node planarization can be completely eliminated. In this planar graph nodes forward in both greedy and perimeter mode and stop at 
junctions where decision about which next road segment to turn into can be determined. [24]

Contention-Based Forwarding (CBF) $[27,41]$ is positionbased unicast forwarding, without neighborhood knowledge. The forwarding decision is based on the actual position of the nodes when packet is forwarded. In position based routing the principle is that the forwarding of the packet, from one node to another node is done based on the local GPS position of the nodes.

Directional Greedy Routing (DGR) uses both position first forwarding and direction first forwarding. Position first forwarding tries to find the closet node towards destination node as the next hop. Direction first forwarding will select the nodes which are moving toward destination node. Among those, the one nearest to the destination will be chosen as next hop. $[33,29]$

DGR uses combination metrics of position-first and direction-first forwarding. The node with largest weighted score among current node itself and its neighbors will be chosen as next hop. If the current node has the largest score, it will carry the packet and forward it later.

Lee introduces a routing solution called "Landmark Overlays for Urban Vehicular Routing Environments" (LOUVRE), an approach that define urban junctions as overlay nodes and create an overlay link if and only if the traffic density of the underlying network guarantees the multi-hop vehicular routing between the two overlay nodes. LOUVRE [31] contains a distributed traffic density estimation scheme to evaluate the presence of an overlay link. Then, efficient routing is performed on the overlay network, guaranteed delivery of packet.

Advanced Greedy Forwarding (AGF) was proposed [32] to combine the speed and direction of a node in the beacon packet and the total travel time from of packet forwarding node to destination node. It is more improved in PDR.

Predictive Direction Greedy Routing (PDGR) predicts the future neighbors. The PDGR calculate the weighted score for current node, its current neighbors and possible future neighbors. It uses two-hop neighbors to generation of possible future neighbors. According to all these weighted scores, next hop is then decided [33]. So PDGR has two parts. First is same as DGR and second is used for future neighbors in a short interval.

In Greedy Routing with Abstract Neighbors Table every node find best route from its $\mathrm{x}$ hop neighborhood. Next forwarding neighbor $\mathrm{E}$ is based on the node $\mathrm{N}$ which is $\mathrm{X}$ hop away from $\mathrm{E}$ and the Destination which has shortest path from $\mathrm{N}$ to $\mathrm{E}$. The smallest matrix for neighbors $\mathrm{E}$ will be selected at the next hop. It is very necessary to select the most promising neighbor as in beacon transmitting $\mathrm{x}$-hop neighbors is too much overhead [35]. It divides the whole map into small areas and has only one representative neighbor per area.

GpsrJ+ minimizes number of hop count by eliminating the unnecessary stops at various junctions of maps. It choose next road segment based on two-hop neighbor beaconing junction which is taken by its neighboring node and predict the next forwarding node. If the next forwarding neighbor node is in different direction then the packet will be forwarded to the junction node. And if is on the same direction then forward to its furthest neighboring node [36]. When it reaches to local maxima then it use the perimeter mode same as GPSR for best forwarding node. if the junction's next node is the best node in the same direction, then it is selected as the best forwarding node; otherwise, the best forwarding node is selected as junction's node.

Street Topology Based Routing update algorithm of ASTAR where junction node have the information about road connectivity. A node at the junction will compute the link up to the next junction and it will find that link is up or down. This junction node is called as master node. Master node will broadcast there link information to other master nodes [37]. It calculates the geographic distance from current node position to the destination nodes, and sends the packet to next node which has less geographic distance.

Greedy Traffic Aware Routing protocol [40] has two parts to search for best route. In Junction's selection, protocol selects the junctions through which the packets must go through. The selection of these junctions is done at runtime and it calculates a traffic density and the curve-metric distance for next junctions when a vehicle wants to forward the packet. The source vehicle or an intermediate vehicle looks for the position of the neighboring junctions using the map. It forwards data between two junctions. As we found the destination junction, the improved greedy strategy is used to forward packets towards the selected junctions. When a packet is received by a node, it forwards the packets to the destination node which is geographically closest. The selection of next node is done on the parameter like velocity, direction and latest position of node and it will be recorded in neighbors table. If the packet reached to local maxima then it will follow the "carry and forward" strategy. The intermediate node carry the packet until it reached to next junction or any other new node comes to its range, As soon as it reached, it will forward the packet.

TOpology-assist Geo-Opportunistic Routing (TO-GO) is a geographic routing protocol which uses the knowledge from via GpsrJ+'s two-hop neighbor information and it select the best node forwarder from the forwarding set between the source and the destination node. The target node is next promising node other than the junction node which is normally chosen by greedy or recovery mode [34].

\section{B. Greedy Perimeter Stateless Routing}

GPSR is an algorithm that consists of greedy packet forwarding and perimeter forwarding. Greedy forwarding is used wherever required, and perimeter forwarding is used in first will not forward.

A source node must know GPS location of destination node for delivery of packets which will route packet to the destination in greedy forwarding mode. In greedy mode, the intermediate forwarding node selects a node as next neighbour if it is closest to destination node. Packets are indicated by their source with their destinations node. As a result, a forwarding node can make a locally optimal, greedy choice in choosing a packet's next hop. An example of greedy next hop choice is shown in Figure 1. Here, intermediate node $x$ receives a packet destined for destination $D$. Dotted circle around $\mathrm{x}$ or any other node denotes range of $\mathrm{x}$, and the arc has radius equal to the distance between $y$ and $D$. As distance between $y$ and $D$ is less than that of between $D$ and any other neighbor's node of $x$. This will be repeated, until the packet reaches $D$. [26]

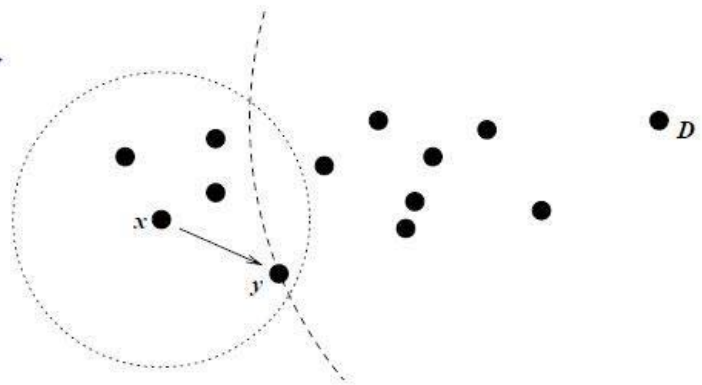

Figure 1 Greedy Forwarding Example 
A beaconing algorithm is used by nodes to know their neighbors positions periodically. Each node broadcast MAC address, its own identifier and position in a beacon. Greedy forwarding's advantage is its dependence only on knowledge of the forwarding node's immediate neighbors. This approach of greedy forwarding has one drawback. A destination node requires a packet move temporarily farther in GPS distance from the destination node to source or intermediate node.

In figure2, an example is shown of a topology where $x$ is near to destination node $D$ than it has its neighbors $w$ and $y$. The dashed arc is radius equal to distance between $\mathrm{D}$ and $\mathrm{x}$. Although it has two paths, $(x \rightarrow y \rightarrow z \rightarrow D)$ and $(x \rightarrow w \rightarrow v \rightarrow D)$ exist between $\mathrm{x}$ and D. $x$ will not choose route using greedy forwarding. Now it will choose to perimeter mode from greedy forwarding mode.

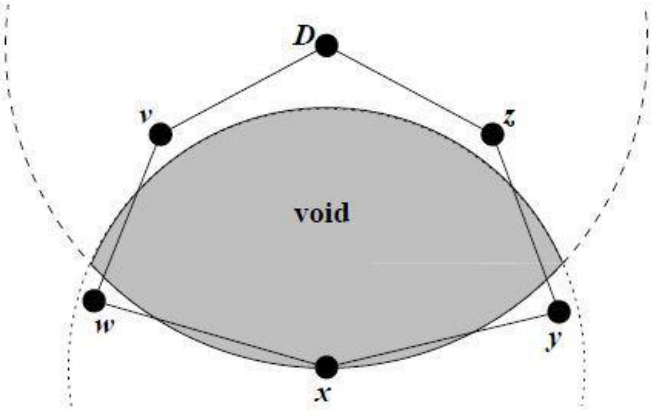

Figure 2 : Void of Node $\mathrm{x}$ with Respect to D.

In perimeter mode next node is selected according to right hand rule and packet is forwarded along to that node towards destination. In figure 2, $x$ will select node according to right hand rule. Mapping perimeters by sending packets on tours of them, using the right-hand rule.

The right hand rule is used for packet routing when any void is present in the network. According to right hand rule, when arriving at node $x$ form node $y$, the next sequential anticlockwise edge will be traversed first.

The drawbacks of greedy forwarding:

- The accuracy reduces if the nodes move due to mobility and packet will loss. It result into packet loss. It is also possible if neighbour table entries is outdated which cause excessive data re-sending.

- Network load will increase due to the beacons

- Link-layer broadcast of the beacons will result in missing of recovery from failure.

This leads to failure in transmission, because nodes being close to each other are not recognized as such.

\section{MFGPSR PROTOCOL}

MFGPSR protocol will start routes searching from source towards destination as per scenario shown in figure 3 .

Source and destination nodes are shown as $\mathrm{S}$ and $\mathrm{D}$ respectively. Initial process is to search for route is shown in Figure 3(a). Source will start broadcasting of packet to all the nodes in range with various information likes Source node, destination node, previous first packet forwarder, hop count, start time and current time. All intermediate node will broadcast same packet as soon as it will be received by them only on first receipt. If same packets received again then it will be discarded by them and will not forward it again.

Reply packets procedure is shown in Figure 3(b). It will start by tracing back path from destination node to source node according to the previous first packet forwarder. At the end of this procedure Source start sending its packet to destination on this path for few packet and repeat from first step to search for new route as mobility of VANET is very high. If packet will not be delivered due to lost in between then also it will start from first step as a part of failure recovery.

MFGPSR Routing Protocol work as below mentioned steps.

Input:

1. Source node $\mathrm{S}$

2. Destination node D

3. Neighbor List $F$

Auxiliary Variables:

1. Initial Packet(S, D, Time)

2. Node Cache(S, D, Node Forwarder, Current Time, Node Backward)

Output:

Done // if Greedy forwarding is successful

Error // if Greedy forwarding is not successful.

Initialization:

Global Average Array $=0$;

$\mathrm{TTL}=1$

Average $=0$

Route Generate Packet ID $=945$

Counter

Begin: MFGPSR Greedy Algorithm

Phase 1: Find Average Density $\mathrm{AVG}_{\mathrm{D}}$

Step 1: Initialize Global Average Array List for all nodes $=0$

Step 2: All neighbors will send Hello packet to all its neighbors' node in range to find total numbers of neighbor's nodes and store it in Global array list.

Step 3: Calculate Average of Global Average Array List and return it.

\section{Phase 2: Route Generate}

Step 4: Calculate $\mathrm{TTL}=\mathrm{C} /\left(1+\mathrm{AVG}_{\mathrm{D}}\right)$.

Step 5: Initial Route Generate Packet will be a broadcasting message from source node with Source Node, Destination Node, Number of Hop Counter and Start time.

Step 6: If all Intermediate nodes will receive Route Generate Packet (945) first time from any node then it will save source node, destination node, previous node forwarder and current time in their cache. Total time after routing should not excess value of TTL.

Step 7: As soon as destination node receives Route Generate Packet, it will reply to packets with backtracking path by selecting previous node which has lowest current time or which is previous node forwarder from its cache until it reach to source node. All intermediate nodes are saving backward node ID with reply.

Step 8: After receiving reply, Route Generate Process will complete.

Phase 3: Packet Forwarding and Route Maintenance 


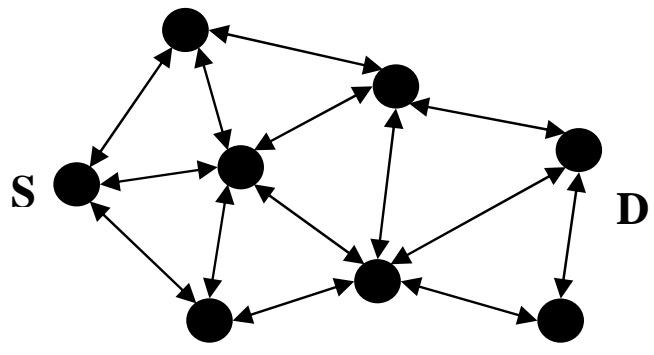

(a)

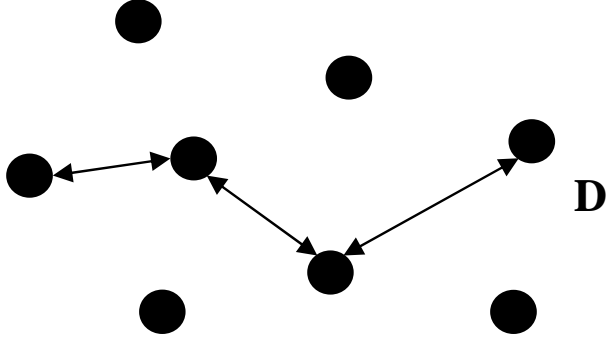

(b)

Figure 3 (a) Packet Transmission from Source to all Possible Nodes (b) Reply with Selected Route

Step 9: After Generating route, source node will start sending packet with packet id $0,1,2, \ldots, 944,0,1, \ldots$.

Step 10: If reply or acknowledgement of packet is not coming from destination node from long period of time then Phase 2 will start again to regenerate Route.

\section{Performance Parameter and Simulaion Result}

In this paper GPSR and MFGPSR is evaluated based on NS-2 network simulator and SUMO traffic simulator. The network dimension used for simulation is $4000 \times 3200$ meter square. The MAC layer protocol used is IEEE 802.11. In this paper comparison is performed against GPSR protocol in Highway, rural and urban scenario. Performance is measured for packet delivery ratio and throughput. Initial location of nodes is obtained using unified location. Sender and receiver nodes are chosen initially for simulation. Any data packets that cannot be delivered to destination due to broken routes are simply dropped. Nodes mobility model used is Random Way Point. We compare the performance of the MFGPSR protocols to several existing protocols like GPCR, GeoDTN+Nav, and GPSR. These map data are transformed into the data format that can be used by ns2. The initial distribution follows the predefined traffic density. Remaining parameters and its value is shown as below.

Table 1: Simulation Parameter

\begin{tabular}{|l|l|}
\hline Parameter & Value \\
\hline Simulation area & $4000 \mathrm{~m} \times 3200 \mathrm{~m}$ \\
\hline \# of intersections & 24 \\
\hline Number of vehicles & $50,150,250$ \\
\hline \# of packet senders & 15 \\
\hline Communication range & $200 \mathrm{~m}$ \\
\hline Vehicle velocity & $5-20$ meter per second \\
\hline Buffer size (in packet) & $\begin{array}{c}10,20,50, \quad 100,200, \\
500, \text { unlimited }\end{array}$ \\
\hline CBR rate & $0.1-1$ packet per \\
& second \\
\hline Data packet size & $10 \mathrm{~B}-4 \mathrm{~KB}$ \\
\hline Beacon interval & $0.5 \mathrm{sec}$ \\
\hline
\end{tabular}

Since GPSR is not proposed for sparsely connected networks, its performance is very poor in VANETs. The nodes are initially placed uniformly at random in a rectangular region. All nodes move according to the random waypoint model, with a maximum velocity of $20 \mathrm{~m} / \mathrm{s}$. We simulate pause times of 200, 400, 600, 800 and 1000 seconds, the highest mobility cases, as they are the most demanding of a routing algorithm.
Comparison of proposed algorithm is done with various protocols for packet delivery ratio on various pause times. Packet delivery ratio of MFGPSR is higher than GPSR and near to GeoDTN+Nav protocol as per shown in Figure 4 for low traffic. As density of node increase to medium traffic, packet delivery ratio increase in modified GPSR and GPCR protocol. But still packet delivery ratio of MFGPSR is better than GPCR. For High traffic density packet delivery ratio of MFGPSR is best amongst all protocol for different pause times.

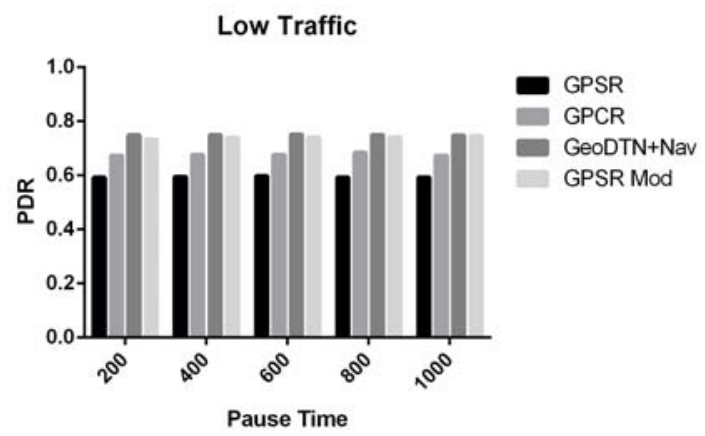

Figure 4 : PDR for different pause time for Low Traffic

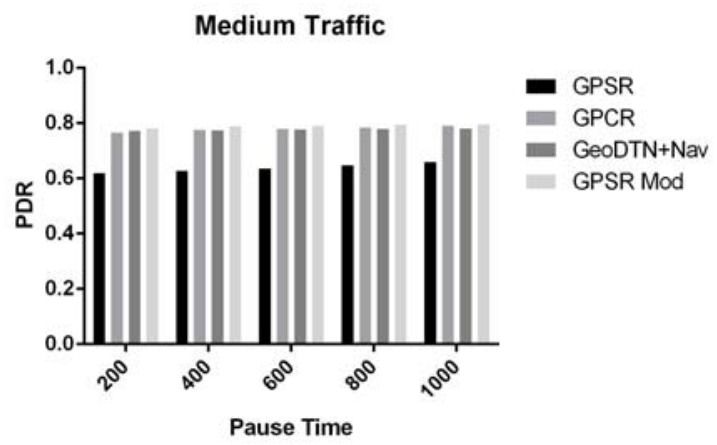

Figure 5 : PDR for different pause time for Medium Traffic

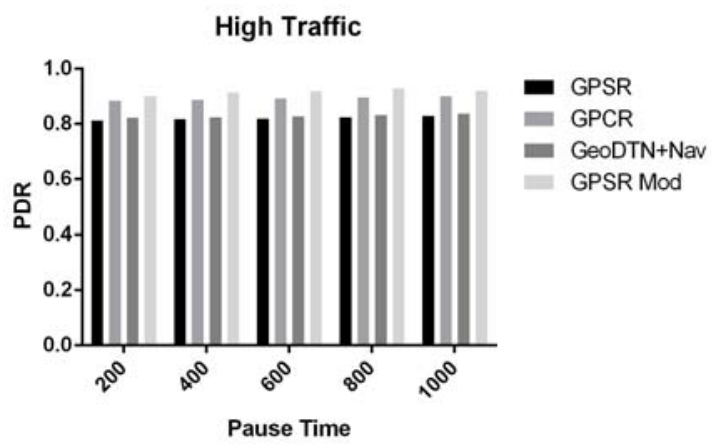

Figure 6 : PDR for different pause time for High Traffic 
Second comparison is performed on no of Hop Count required to transmit packet to destination node against total number of nodes in network. Packet delivery ratio of GeoDTN+Nav is better while its Hop count is higher in GeoDTN+Nav. MFGPSR's Hop count is almost same as GPSR protocol.

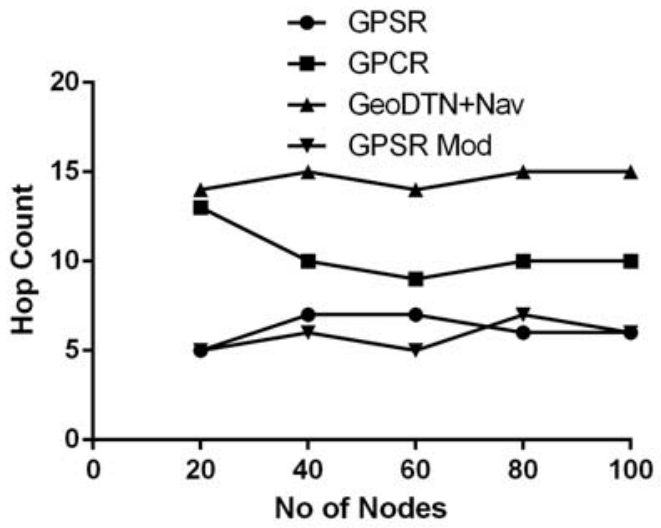
nodes

Figure 7 : Number of Hop Count for different numbers of

\section{Conclusions}

This paper proposes Enhanced Route Selection Approach of GPSR using Multipath Forwarding Approach in VANET. We have presented modified GPSR protocol that uses better greedy approach than geography location to achieve better packet delivery ratio with maintained hop count as compared to all other protocol. Modified GPSR consistently deliver packets at rate of around $87 \%$ on various traffic scenarios. As the number of node increase packet delivery ratio is also increasing. Finally modified GPSR keeps propositional Hop count for different no of neighbour nodes. From given result, it is clear that proposed algorithms generate good packet delivery ratio amongst all available algorithms.

\section{FUTURE WORK}

Proposed algorithm works with new greedy approach on existing GPSR protocol, which can be extended along with geography location on various traffic data as well as delay tolerant or non delay tolerant networks. Proposed algorithm could be checked against various other performance criteria or various sizes of maps.

\section{ACKNOWLEDGMENT}

We thank Dr. Manish Chaturvedi for providing guidance for research work and simulation tool.

\section{VIII.REFERENCES}

[1] Bujari, A., Maggiorini, D., Palazzi, C.E., Quadri, C., \& Rossi, G.P. (2012). A solution for mobile DTN in a real urban scenario. 2012 IEEE Wireless Communications and Networking Conference Workshops (WCNCW), 344-349.

[2] Kumar, S., Ahmed, S. H., Qasim, U., Khan, Z. A., Amjad, N., Azeem, M. Q., ... \& Javaid, N. (2014). Analyzing link and path availability of routing protocols in vehcular ad-hoc networks. Journal of Basic and Applied Scientific Research, 4(2), 189-206.

[3] Xing, K., Wu, W., Ding, L., Wu, L., \& Willson, J. (2014). An efficient routing protocol based on consecutive forwarding prediction in delay tolerant networks. International Journal of Sensor Networks, 15(2), 73-82.

[4] Yu, D., \& Ko, Y. B. (2009, February). FFRDV: fastest-ferry routing in DTN-enabled vehicular ad hoc networks. In Advanced Communication Technology, 2009. ICACT 2009. 11th International Conference on (Vol. 2, pp. 1410-1414). IEEE.

[5] Panchal, J., Patel, H., (2015). A Review on Multi-Hop Message Dissemination Strategies in VANET Environment, International Journal for Scientific Research \& Development (Vol-3(1), pp803)

[6] Wu, D., Zhu, G., \& Zhao, D. (2013). Adaptive carry-store forward scheme in two-hop vehicular delay tolerant networks. IEEE Communications Letters, 17(4), 721-724.

[7] Khabbaz, M. J., Fawaz, W. F., \& Assi, C. M. (2011). Probabilistic bundle relaying schemes in two-hop vehicular delay tolerant networks. IEEE Communications Letters, 15(3), 281-283.

[8] Al Hanbali, A., Ibrahim, M., Simon, V., Varga, E., \& Carreras, I. (2008, October). A survey of message diffusion protocols in mobile ad hoc networks. In Proceedings of the 3rd International Conference on Performance Evaluation Methodologies and Tools (p. 82). ICST (Institute for Computer Sciences, SocialInformatics and Telecommunications Engineering).

[9] Burgess, J., Gallagher, B., Jensen, D. D., \& Levine, B. N. (2006, April). MaxProp: Routing for Vehicle-Based DisruptionTolerant Networks. In Infocom.

[10] Makwana, K., Patel, H., (2015). Survey Paper on Proactive Topology Based Routing Protocol in VANET, International Journal for Scientific Research \& Development (Vol-3(3), pp846).

[11] Cheng, P. C., Lee, K. C., Gerla, M., \& Härri, J. (2010). GeoDTN+ Nav: geographic DTN routing with navigator prediction for urban vehicular environments. Mobile Networks and Applications, 15(1), 61-82.

[12] Leguay, J., Friedman, T., \& Conan, V. (2005). Evaluating mobility pattern space routing for DTNs.arXiv preprint cs/0511102.

[13] Moustafa, H., \& Zhang, Y. (2009). Vehicular networks: techniques, standards, and applications. Auerbach publications.

[14] Leontiadis, I., \& Mascolo, C. (2007, June). GeOpps: Geographical opportunistic routing for vehicular networks. In World of Wireless, Mobile and Multimedia Networks, 2007. WoWMoM 2007. IEEE International Symposium on a (pp. 1-6). Ieee.

[15] Trivedi, B., Patel, H., (2015). Survey Paper on Reactive (ondemand)-Topology Based Routing Protocol in VANET, International Journal of Computer Science \& Engineering Technology (Vol-6(2), pp48-52).

[16] Zhao, J., \& Cao, G. (2008). VADD: Vehicle-assisted data delivery in vehicular ad hoc networks. IEEE transactions on vehicular technology, 57(3), 1910-1922.

[17] Lo, W. Z., Gao, J. S., \& Lo, S. C. (2012, May). Distance-aware routing with copy control in vehicle-based DTNs. In Vehicular Technology Conference (VTC Spring), 2012 IEEE 75th (pp. 15). IEEE.

[18] Fu, Q., Zhang, L., Feng, W., \& Zheng, Y. (2011, September). Dawn: A density adaptive routing algorithm for vehicular delay tolerant sensor networks. In Communication, Control, and Computing (Allerton), 2011 49th Annual Allerton Conference on (pp. 1250-1257). IEEE.

[19] Trivedi, B., Patel, H., (2015). Enhancement in AODV-reactive topology based routing protocol in VANET", International Journal for Scientific Research \& Development (Vol-3(8), pp469).

[20] Soares, V. N., Rodrigues, J. J., \& Farahmand, F. (2014). GeoSpray: A geographic routing protocol for vehicular delaytolerant networks. Information Fusion, 15, 102-113. 
[21] Lochert, C., Hartenstein, H., Tian, J., Fussler, H., Hermann, D., \& Mauve, M. (2003, June). A routing strategy for vehicular ad hoc networks in city environments. In Intelligent Vehicles Symposium, 2003. Proceedings. IEEE(pp. 156-161). IEEE.

[22] Seet, B. C., Liu, G., Lee, B. S., Foh, C. H., Wong, K. J., \& Lee, K. K. (2004, May). A-STAR: A mobile ad hoc routing strategy for metropolis vehicular communications. In International Conference on Research in Networking (pp. 989-999). Springer, Berlin, Heidelberg.

[23] Naumov, V., \& Gross, T. R. (2007, May). Connectivity-aware routing (CAR) in vehicular ad-hoc networks. In INFOCOM 2007. 26th IEEE International Conference on Computer Communications. IEEE (pp. 1919-1927). IEEE.

[24] Lochert, C., Mauve, M., Füßler, H., \& Hartenstein, H. (2005). Geographic routing in city scenarios. ACM SIGMOBILE mobile computing and communications review, 9(1), 69-72.

[25] Upadhyay, R., Patel, H., (2015). AODV Routing Protocol in VANETs in Comparison with OLSR and DSR, International Journal for Scientific Research \& Development (Vol-2(11), pp329).

[26] Karp, B., \& Kung, H. T. (2000, August). GPSR: Greedy perimeter stateless routing for wireless networks. In Proceedings of the 6th annual international conference on Mobile computing and networking (pp. 243-254). ACM.

[27] Fussler, H. O. L. G. E. R., Widmer, J., Mauve, M., \& Hartenstein, H. (2003, October). A novel forwarding paradigm for position-based routing (with implicit addressing). In Computer Communications, 2003. CCW 2003. Proceedings. 2003 IEEE 18th Annual Workshop on (pp. 194-200). IEEE.

[28] Adler, C., J., (2006), Information dissemination in vehicular ad hoc networks, Master's thesis, University of Munich, April 2006

[29] Prasanth, K., Duraiswamy, D. K., Jayasudha, K., \& Chandrasekar, D. C. (2010). Improved packet forwarding approach in Vehicular ad hoc networks using RDGR algorithm. arXiv preprint arXiv:1003.5437.

[30] Moustafa, H., \& Zhang, Y. (2009). Vehicular networks: techniques, standards, and applications. Auerbach publications.

[31] Lee, K. C., Le, M., Harri, J., \& Gerla, M. (2008, September). Louvre: Landmark overlays for urban vehicular routing environments. In Vehicular Technology Conference, 2008. VTC 2008-Fall. IEEE 68th (pp. 1-5). IEEE.
[32] Naumov, V., Baumann, R., \& Gross, T. (2006, May). An evaluation of inter-vehicle ad hoc networks based on realistic vehicular traces. In Proceedings of the 7th ACM international symposium on Mobile ad hoc networking and computing (pp. 108-119). ACM.

[33] Gong, J., Xu, C. Z., \& Holle, J. (2007, June). Predictive directional greedy routing in vehicular ad hoc networks. In Distributed Computing Systems Workshops, 2007. ICDCSW'07. 27th International Conference on (pp. 2-2). IEEE.

[34] Lee, K. C., Lee, U., \& Gerla, M. (2009, February). TO-GO: TOpology-assist geo-opportunistic routing in urban vehicular grids. In Wireless On-Demand Network Systems and Services, 2009. WONS 2009. Sixth International Conference on (pp. 1118). IEEE.

[35] Schnaufer, S., \& Effelsberg, W. (2008, June). Position-based unicast routing for city scenarios. In World of Wireless, Mobile and Multimedia Networks, 2008. WoWMoM 2008. 2008 International Symposium on a (pp. 1-8). IEEE.

[36] Lee, K. C., Härri, J., Lee, U., \& Gerla, M. (2007, November). Enhanced perimeter routing for geographic forwarding protocols in urban vehicular scenarios. In Globecom Workshops, 2007 IEEE (pp. 1-10). IEEE.

[37] Forderer, D., (2005). Street-Topology Based Routing. Master's thesis, University of Mannheim.

[38] Lin, Y. W., Chen, Y. S., \& Lee, S. L. (2010). Routing Protocols in Vehicular Ad Hoc Networks: A Survey and Future Perspectives. J. Inf. Sci. Eng., 26(3), 913-932.

[39] Jerbi, M., Senouci, S. M., Meraihi, R., \& Ghamri-Doudane, Y. (2007, June). An improved vehicular ad hoc routing protocol for city environments. In Communications, 2007. ICC'07. IEEE International Conference on (pp. 3972-3979). IEEE.

[40] Jerbi, M., Meraihi, R., Senouci, S. M., \& Ghamri-Doudane, Y. (2006, September). GyTAR: improved greedy traffic aware routing protocol for vehicular ad hoc networks in city environments. In Proceedings of the 3rd international workshop on Vehicular ad hoc networks (pp. 88-89). ACM.

[41] Füßler, H., Widmer, J., Käsemann, M., Mauve, M., \& Hartenstein, H. (2003). Contention-based forwarding for mobile ad hoc networks. Ad Hoc Networks, 1(4), 351-369. 